\title{
Dihedral Groups as Epimorphic Images of Some Fibonacci Groups
}

\author{
Abdullahi Umar ${ }^{a^{*}}$ and Bashir Ali
}

${ }^{a}$ Department of Mathematics and Statistics, Sultan Qaboos University,Al-Khod, PC 123 - Oman, ${ }^{*}$ EEmail: aumarh@squ.edu.om. ${ }^{b}$ Department of Mathematics and Computer Science, Nigerian Defence Academy, Kaduna - Nigeria.

ABSTRACT: The Fibonacci groups are defined by the presentation $F(r, n)=$ $\left\langle a_{1}, a_{2}, \ldots, a_{n}: a_{1} a_{2} \cdots a_{r}=a_{r+1}, a_{2} a_{3} \cdots a_{r+1}=a_{r+2}, \ldots, a_{n} a_{1} \cdots a_{r-1}=a_{r}\right\rangle$, where $r>0$, $n>0$ and all subscripts are assumed to be reduced modulo $n$. In this paper we give an alternative proof that for $r \geq 0, F(2 r, 4 r+2), F(4 r+3,8 r+8)$ and $F(4 r+5,8 r+12)$ are all infinite by establishing a morphism (or group homomorphism) onto the dihedral group $D_{n}$ for all $n>2$.

Keywords: Group; Fibonacci group; Dihedral group; (homo) Morphism.

$$
\begin{aligned}
& \text { مجموعات دايهيدرل كصورة متماثلة لمجموعات فييوناتثىى } \\
& \text { عبدالله عمر و بثير علي } \\
& \text { ملخص : تعرّف مجمو عات فييوناتثي تعرف بو اسطة التمثيل } \\
& F(r, n)=\left\langle a_{1}, a_{2}, \ldots, a_{n}: a_{1}, a_{2}, a_{3} \ldots a_{r+1}=a_{r+2}, \ldots, a_{n} a_{1} \ldots a_{r-1}=a_{r}\right\rangle \\
& \text { عندما تكون }
\end{aligned}
$$

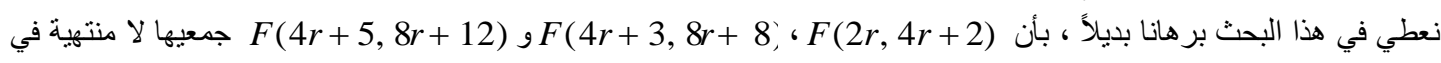

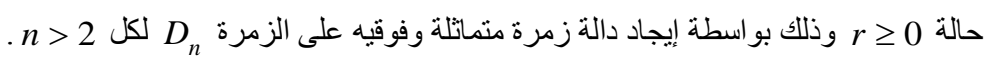

$$
\begin{aligned}
& \text { مفتاح الكلمات : مجمو عات ، مجمو عات فييوناتثي ، مجمو عات دايهيدرل ، تنشابه شكلي. }
\end{aligned}
$$

\section{Introduction}

For $r \geq 1$ and $n \geq 1$ the Fibonacci group $F(r, n)$ is defined by the presentation:

$$
F(r, n)=\left\langle a_{1}, a_{2}, \ldots, a_{n}: a_{1} a_{2} \cdots a_{r}=a_{r+1}, a_{2} a_{3} \cdots a_{r+1}=a_{r+2}, \cdots, a_{n} a_{1} \cdots a_{r-1}=a_{r}\right\rangle,
$$

where all subscripts are assumed to be reduced modulo $n$, if necessary. These groups were first introduced by Conway (1965) and have been studied over the last few decades. For a nice survey article see (Thomas, 1991) or (Campbell et al., 1992).

The dihedral group of order $2 n$ denoted by $D_{n}$ is usually defined by

$$
D_{n}=\left\langle x, y: x^{n}=y^{2}=1, y x^{-1}=x y\right\rangle .
$$

It is well known that $x$ and $y$ in $D_{n}$ satisfy the relations summarized in the next lemma.

Lemma 1.1 For all $0 \leq k \leq n-1$ we have

${ }^{1}$ MSC2010 : $20 \mathrm{~F} 05$ 


\section{FIBONACCI GROUPS}

(a) $x^{-k}=x^{n-k}$;

(b) $y^{-1}=y$;

(c) $y x^{k}=x^{n-k} y$;

(d) $\left(x^{k} y\right)^{2}=1$;

(e) $x^{k} y x^{k}=y$;

(f) $y x^{k} y=x^{n-k}$.

Thus we may write the elements of $D_{n}$ uniquely as $x^{k}$ or $x^{k} y$ for $k=0,1,2, \ldots, n-1$.

Campbell et al. (2004) explored the connection between the Fibonacci groups and finite groups via the concept of Fibonacci length. In the case where the finite groups were dihedral they obtained satisfactory results. In this note we further explore the connection between the Fibonacci groups and dihedral groups in a different manner. In particular, we establish epimorphisms between Fibonacci groups in certain classes and all finite dihedral groups of order greater than 4, thus giving alternative proofs regarding the infiniteness of the groups in these classes of Fibonacci groups. For basic concepts in group theory we refer the reader to (Gallian, 1998). The following lemma for $F(r, n)$ is indispensable for our discourse.

Lemma 1.2 For all $r>0$ and $m \geq 2$ we have $a_{m+r}=a_{m-1}^{-1} a_{m+r-1}^{2}$ in $F(r, n)$.

Proof.

$$
\begin{aligned}
a_{m+r} & =a_{m} a_{m+1} \cdots a_{m+r-1}=a_{m-1}^{-1}\left(a_{m-1} a_{m} a_{m+1} \cdots a_{m+r-2}\right) a_{m+r-1} \\
\quad= & a_{m-1}^{-1} a_{m+r-1}^{2} .
\end{aligned}
$$

\section{Morphic Images}

First we consider the Fibonacci groups $F(2 r, 4 r+2)$.

Theorem 2.1 Let $r>0$. There exist morphisms from $F(2 r, 4 r+2)$ onto $D_{n}$ for all $n \geq 3$. Hence $F(2 r, 4 r+2)$ is infinite.

We are going to prove this theorem via a sequence of lemmas. However, we first define a mapping from the first $2 r$ generators of $F(2 r, 4 r+2)$ onto the generators of $D_{n}$ by

$$
a_{x} \mapsto x \text { and } a_{i} \mapsto y(i=2,3, \ldots, 2 r) .
$$

Then the next lemma gives the images of the remaining generators: $a_{2 r+1}, a_{2 r+2}, \cdots, a_{4 r+1}$.

\section{Lemma 2.2}

(a) $a_{2 r+1} \mapsto x y(r \geq 1)$;

(b) $a_{2 r+2} \mapsto x^{n-1}(r \geq 1)$;

(c) $a_{2 r+3} \mapsto x^{2} y(r \geq 1)$;

(d) $a_{2 r+i} \mapsto y(r \geq 2$ and $4 \leq i \leq 2 r+1)$.

Proof. Using Lemma 1.2 we see that

(a) $a_{2 r+1}=a_{1} a_{2} \cdots a_{2 r} \mapsto x y^{2 r-1}=x y(r \geq 1)$;

(b) $a_{2 r+2}=a_{1}^{-1} a_{2 r+1}^{2} \mapsto x^{-1}(x y)^{2}=x^{n-1}(r \geq 1)$;

(c) $a_{2 r+3}=a_{2}^{-1} a_{2 r+2}^{2} \mapsto y^{-1}\left(x^{n-1}\right)^{2}=x^{2} y(r \geq 1)$;

(d) This proof is by induction.

Basis step: By Lemma 1.2 and (c) above, we see that

$$
a_{2 r+4}=a_{3}^{-1} a_{2 r+3}^{2} \mapsto y^{-1}\left(x^{2} y\right)^{2}=y .
$$

Inductive step: Suppose that $a_{2 r+i} \mapsto y$ (for some $4 \leq i \leq 2 r$ ). Using Lemma 1.2 again we see that

$$
a_{2 r+i+1}=a_{i}^{-1} a_{2 r+i}^{2} \mapsto y^{-1} y^{2}=y,
$$


as required.

Lemma 2.3 For $r \geq 1$ we have

(a) $a_{4 r+2} \mapsto x y(r \geq 1)$;

(b) $a_{1}=a_{4 r+3} \mapsto x$;

(c) $a_{i-2}=a_{4 r+i} \mapsto y(4 \leq i \leq 2 r+2)$.

Proof. Using Lemmas 1.2 and 2.2 we see that

(a) $a_{4 r+2}=a_{2 r+1}^{-1} a_{4 r+1}^{2} \mapsto(x y)^{-1} y^{2}=x y$;

(b) $a_{4 r+3}=a_{2 r+2}^{-1} a_{4 r+2}^{2} \mapsto x(x y)^{2}=x$;

(c) This proof is by induction.

Basis step: By Lemma 1.2 and (b) above, we see that

$$
a_{4 r+4}=a_{2 r+3}^{-1} a_{4 r+3}^{2} \mapsto\left(x^{2} y\right)^{-1} x^{2}=y .
$$

Inductive step: Suppose that $a_{4 r+i} \mapsto y$ (for some $4 \leq i \leq 2 r+1$ ). Using Lemma 1.2 again we see that

as required.

$$
a_{4 r+i+1}=a_{2 r+i}^{-1} a_{4 r+i}^{2} \mapsto y^{-1} y^{2}=y,
$$

It is now clear from Lemmas 2.2 and 2.3 that the mapping defined in (2) is indeed a morphism onto $D_{n}$, which preserves all the relations of $F(2 r, 4 r+2)$ and so Theorem 2.1 is proved.

Next we consider the Fibonacci groups $F(4 r+3,8 r+8)$.

Theorem 2.4 Let $r \geq 0$. There exist morphisms from $F(4 r+3,8 r+8)$ onto $D_{n}$ for all $n \geq 3$. Hence $F(4 r+3,8 r+8)$ is infinite.

As in the previous case, we are going to prove this theorem via a sequence of lemmas. First, we define a mapping from the first $4 r+3$ generators of $F(4 r+3,8 r+8)$ onto the generators of $D_{n}$ by

$$
a_{i}, a_{2 r+3} \mapsto x \text { and } a_{i} \mapsto y,
$$

where $2 \leq i \leq 4 r+3, i \neq 2 r+3$ and $r \geq 0$. Then the next two lemmas give the images of the remaining generators: $a_{4 r+4}, a_{4 r+5}, \cdots, a_{8 r+8}$.

Lemma 2.5 For $r \geq 0$ we have
(a) $a_{4 r+4} \mapsto y$
(b) $a_{4 r+5} \mapsto x^{n-1}$;
(c) $a_{4 r+6} \mapsto x^{2} y$;
(d) $a_{4 r+i} \mapsto y(7 \leq i \leq 2 r+6)$.

Proof. Using Lemma 1.2 we see that
(a) $a_{4 r+4}=a_{1} a_{2} \cdots a_{4 r+3} \mapsto x y^{2 r+1} x y^{2 r}=y$;
(b) $a_{4 r+5}=a_{1}^{-1} a_{4 r+4}^{2} \mapsto x^{-1} y^{2}=x^{n-1}$;
(c) $a_{4 r+6}=a_{2}^{-1} a_{4 r+5}^{2} \mapsto y^{-1}\left(x^{n-1}\right)^{2}=x^{2} y$;
(d) This proof is by induction.

Basis step: By Lemma 1.2 and (c) above, we see that

$$
a_{4 r+7}=a_{3}^{-1} a_{4 r+6}^{2} \mapsto y^{-1}\left(x^{2} y\right)^{2}=y .
$$

Inductive step: Suppose that $a_{4 r+i} \mapsto y$ (for some $7 \leq i \leq 2 r+5$ ). Using Lemma 1.2 and the induction 
hypothesis we see that

as required.

$$
a_{4 r+i+1}=a_{i-3}^{-1} a_{4 r+i}^{2} \mapsto y^{-1} y^{2}=y,
$$

Lemma 2.6 For $r \geq 0$ we have

(a) $a_{6 r+7} \mapsto x^{n-1}$;

(b) $a_{6 r+8} \mapsto x^{2} y$;

(c) $a_{6 r+i} \mapsto y(9 \leq i \leq 2 r+8)$.

Proof. Using Lemmas 1.2 and 2.5 we see that

(a) $a_{6 r+7}=a_{2 r+3}^{-1} a_{6 r+6}^{2} \mapsto x^{-1} y^{2}=x^{n-1}$;

(b) $a_{6 r+8}=a_{2 r+4}^{-1} a_{6 r+7}^{2} \mapsto y^{-1}\left(x^{n-1}\right)^{2}=x^{2} y$;

(c) This proof is by induction.

Basis step: For $i=9$, we see tha

$$
a_{6 r+9}=a_{2 r+5}^{-1} a_{6 r+8}^{2} \mapsto y^{-1}\left(x^{2} y\right)^{2}=y .
$$

Inductive step: Suppose that $a_{6 r+i} \mapsto y$ (for some $9 \leq i \leq 2 r+7$ ). Then using Lemma 1.2, the fact that $i \geq 9$ and induction hypothesis we see that

$$
a_{6 r+i+1}=a_{(2 r-3)+i}^{-1} a_{6 r+i}^{2} \mapsto y^{-1} y^{2}=y,
$$

as required.

Lemma 2.7 for $r \geq 0$ we have

(a) $a_{1}=a_{8 r+9} \mapsto x$;

(b) $a_{i-8}=a_{8 r+i} \mapsto y(10 \leq i \leq 2 r+10)$.

Proof. Using Lemmas 1.2, 2.5 and 2.6 we see that

(a) $a_{8 r+9}=a_{4 r+5}^{-1} a_{8 r+8}^{2} \mapsto\left(x^{n-1}\right)^{-1} y^{2}=x$;

(b) for $10 \leq i \leq 2 r+10$, we use induction.

Basis step: For $i=10$, we see that

$$
a_{(8 r+8)+2}=a_{4 r+6}^{-1} a_{(8 r+8)+1}^{2} \mapsto\left(x^{2} y\right)^{-1} x^{2}=y .
$$

Inductive step: Suppose that $a_{8 r+i} \mapsto y$ (for some $10 \leq i \leq 2 r+9$ ). Then using Lemma 1.2, (a) above and the induction hypothesis we see that

as required.

$$
a_{8 r+i+1}=a_{4 r+i-3}^{-1} a_{8 r+i}^{2} \mapsto y^{-1} y^{2}=y
$$

Lemma 2.8 For $r \geq 0$ we have

(a) $a_{2 r+3}=a_{8 r+(2 r+11)} \mapsto x$;

(b) $a_{i-8}=a_{8 r+i} \mapsto y(2 r+12 \leq i \leq 4 r+11)$.

Proof. Using Lemmas 1.2, 2.6 and 2.7 we see that

(a) $a_{8 r+(2 r+11)}=a_{4 r+(2 r+7)}^{-1} a_{8 r+(2 r+10)}^{2} \mapsto\left(x^{n-1}\right)^{-1} y^{2}=x$;

(b) for $2 r+12 \leq i \leq 4 r+11$ we use induction.

Basis step: For $i=2 r+12$, we see that

$$
a_{8 r+(2 r+12)}=a_{4 r+(2 r+8)}^{-1} a_{8 r+(2 r+11)}^{2} \mapsto\left(x^{2} y\right)^{-1} x^{2}=y .
$$


Inductive step: Suppose that $a_{8 r+i} \mapsto y$ (for some $2 r+12 \leq i \leq 4 r+10$ ). Then using Lemma 1.2 (a) above and the induction hypothesis we see that

$$
a_{8 r+i+1}=a_{4 r+i-3}^{-1} a_{8 r+i}^{2} \mapsto y^{-1} y^{2}=y,
$$

as required.

It is now clear from Lemmas 2.5, 2.6, 2.7 and 2.8 that the mapping defined in (3) is indeed a morphism onto $D_{n}$, which preserves all the relations of $F(4 r+3,8 r+8)$ and so Theorem 2.4 is proved.

Finally we consider the Fibonacci groups $F(4 r+5,8 r+12)$.

Theorem 2.9 Let $r \geq 0$. There exist morphisms from $F(4 r+5,8 r+12)$ onto $D_{n}$ for all $n \geq 3$. Hence $F(4 r+5,8 r+12)$ is infinite.

As in the previous cases, we are going to prove this theorem via a sequence of lemmas. However, since the proofs are similar to the previous case we are going to state the corresponding results without proofs. We first define a mapping from the first $4 r+5$ generators of $F(4 r+5,8 r+12)$ onto the generators of $D_{n}$ by

$$
a_{1}, a_{2 r+3} \mapsto x \text { and } a_{i} \mapsto y
$$

where $2 \leq i \leq 4 r+5, i \neq 2 r+3$ and $r \geq 0$. Analogously to Lemma 2.5 we have

Lemma 2.10 For $r \geq 0$
(a) $a_{4 r+6} \mapsto y$;
(b) $a_{4 r+7} \mapsto x^{n-1}$;
(c) $a_{4 r+8} \mapsto x^{2} y$;
(d) $a_{4 r+i} \mapsto y(9 \leq i \leq 2 r+8)$.

Analogously to Lemma 2.6 we have

Lemma 2.11 For $r \geq 0$ we have.

(a) $a_{6 r+9} \mapsto x^{n-1}$;

(b) $a_{6 r+10} \mapsto x^{2} y$;

(c) $a_{6 r+i} \mapsto y(11 \leq i \leq 2 r+12)$.

Analogously to Lemma 2.7 we have

Lemma 2.12 For $r \geq 0$ we have

(a) $a_{1}=a_{8 r+13} \mapsto x$

(b) $a_{i-12}=a_{8 r+i} \mapsto y(14 \leq i \leq 2 r+14)$.

Analogously to Lemma 2.8 we have

Lemma 2.13 For $r \geq 0$ we have

(a) $a_{2 r+3}=a_{8 r+(2 r+15)} \mapsto x$;

(b) $a_{i-12}=a_{8 r+i} \mapsto y(2 r+16 \leq i \leq 4 r+17)$.

It is now clear from Lemmas 2.10, 2.11, 2.12 and 2.13 that the mapping defined in (4) is indeed a morphism onto $D_{n}$, which preserves all the relations of $F(4 r+5,8 r+12)$ and so Theorem 2.9 is proved. 


\section{FIBONACCI GROUPS}

\section{Acknowledgements}

Bashir Ali acknowledges the support and hospitality of Sultan Qaboos University in Spring 2011 during which period this work was completed. Bashir Ali also thanks the Nigerian Defence Academy for research leave and ETF Nigeria for financial support. We also thank the referees for helpful comments and suggestions, which greatly improved the exposition of this paper.

\section{References}

CAMPBELL, C.M., DOOSTIE, H. and ROBERTSON, E.F. 2004. On the Fibonacci Length of Powers of Dihedral Groups. Applications of Fibonacci Numbers 9, Ed. F.T. Howard, Kluwer, Dordrecht, 69-85.

CAMPBELL, C.M., ROBERTSON, E.F. and THOMAS, R.M. 1992. Fibonacci Groups and Semigroups. Technical Report CSD-50, Department of Computing Studies, University of Leicester.

CONWAY, J.H. 1965. Solution of Advanced Problem 5327. American Mathematical Monthly 72(8): 915.

GALLIAN, J.A. 1998. Contemporary Abstract Algebra, Houghton Mifflin, Boston/New York.

THOMAS, R.M. 1991. The Fibonacci groups revisited. In Proceedings of Groups - St Andrews 1989, Volume 2 (London Math. Soc. Lecture Note Series 160, Cambridge University Press, 1991). (Eds.) CAMPBELL, C.M. and ROBERTSON, E.F. 445-454.

Received 12 April 2011

Accepted 16 June 2012 